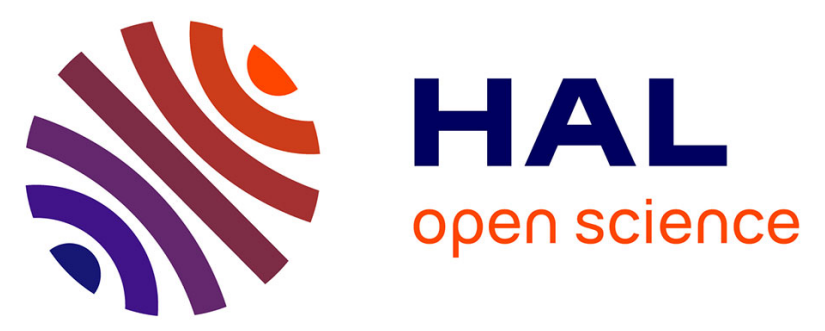

\title{
Combined TD-DFT-SOS-CIS(D) Study of BOPHY Derivatives with Potential Application in Biosensing
}

Miguel-Armando Ponce-Vargas, Cloé Azarias, Denis Jacquemin, Boris Le Guennic

\section{- To cite this version:}

Miguel-Armando Ponce-Vargas, Cloé Azarias, Denis Jacquemin, Boris Le Guennic. Combined TDDFT-SOS-CIS(D) Study of BOPHY Derivatives with Potential Application in Biosensing. Journal of Physical Chemistry B, 2017, 121 (48), pp.10850-10858. 10.1021/acs.jpcb.7b09698 . hal-01660897

\section{HAL Id: hal-01660897 https://u-bourgogne.hal.science/hal-01660897}

Submitted on 2 Mar 2018

HAL is a multi-disciplinary open access archive for the deposit and dissemination of scientific research documents, whether they are published or not. The documents may come from teaching and research institutions in France or abroad, or from public or private research centers.
L'archive ouverte pluridisciplinaire HAL, est destinée au dépôt et à la diffusion de documents scientifiques de niveau recherche, publiés ou non, émanant des établissements d'enseignement et de recherche français ou étrangers, des laboratoires publics ou privés. 


\title{
A Combined TD-DFT-SOS-CIS(D) Study of
}

\section{BOPHY Derivatives with Potential Application in}

\section{Biosensing}

\author{
Miguel Ponce-Vargas ${ }^{* a, b}$, Cloé Azarias $^{c}$, Denis Jacquemin ${ }^{c, d}$, and Boris Le Guennic ${ }^{b}$. \\ ${ }^{a}$ Université Paris-Est, Laboratoire Modélisation et Simulation Multi-Échelle, MSME UMR 8208 \\ CNRS, 5 bd Descartes, 77454 Marne-la-Vallée, France. \\ miguel.poncevargas@u-pem.fr \\ b Institut des Sciences Chimiques de Rennes, UMR 6226 CNRS-Université de Rennes 1, 263 \\ Avenue du Général Leclerc, 35042 Rennes Cedex, France. \\ ${ }^{\mathrm{c}}$ Laboratoire CEISAM, UMR CNRS 6230, Université de Nantes, 2 Rue de la Houssinière, BP \\ 92208, 44322 Nantes Cedex 3, France \\ ${ }^{\mathrm{d}}$ Institut Universitaire de France, 1 rue Descartes, 75005 Paris Cedex 05, France.
}

\begin{abstract}
A set of thirteen BOPHY dyes is studied through a hybrid TD-DFT-SOS-CIS(D) approach accounting for solvent effects, in order to shed light onto the structure-property relationships of these recently developed chromophores. In a first step, we calculate the absorption fluorescence crossing points with refined TD-DFT models considering the influences of both vibrational and
\end{abstract}


solvent contributions. We found that the systematic overestimation of the $0-0$ energies is effectively reduced by combining PCM-TD-DFT with a scaled opposite spin-configuration interaction singles with a double correction [SOS-CIS(D)]. Next, for a representative system, the vibrationally resolved spectrum within the harmonic approximation is computed on the basis of TD-DFT vibrational signatures and an excellent match with experiment is found. Finally, the influence of different lateral groups on the spectroscopic properties is rationalized by investigating charge transfer parameters and examining electronic density difference maps. It is found that one can tune the position of the absorption/emission maxima by a judicious choice of the lateral substituents or by using $\pi$-extended segments. The largest absorption and emission wavelengths as well as the largest Stokes shifts are obtained for BOPHYs containing strong electron-donor dimethylaminophenyl groups attached to the $\alpha$-positions of the pyrrole units through vinyl linkers, making these chromophores promising candidates for bioluminescence applications. 


\section{INTRODUCTION}

During the past decades, technological advances in the field of bioimaging have allowed monitoring several intracellular processes that were previously invisible to human eyes ${ }^{1-3}$. In this framework, the search for novel molecules exhibiting appropriate optical signatures for fluorescent probe applications, i.e., high fluorescent quantum yield, photo and chemical stability, biocompatibility with the medium, and large Stokes shift, has been particularly intense ${ }^{4-7}$. The strong UV-absorption and high fluorescence quantum-yield of BODIPY (4,4-difluoro-4-bora3a,4a-diaza-s-indocene) dyes have prompted several research groups to synthesize structurally similar compounds that may present enhanced luminescent properties ${ }^{8,9}$. Among these new fluorophores, bis(difluoroboron)-1,2-bis((pyrrol-2-yl)methylene)hydrazine $\quad$ (BOPHY, see Scheme 1) derivatives, that contain two borondifluoride units bridged by a coupled pair of Schiff bases, arise as promising candidates as they exhibit large extinction coefficients ${ }^{10}$, emission quantum yields close to unity ${ }^{10}$ and deep-red fluorescence emission ${ }^{11-13}$. Since their discovery, several structural modifications of BOPHYs have been conducted in order to boost their emissive properties, including the incorporation of halogens ${ }^{14}$, perylene ${ }^{14}$, and ferrocene units ${ }^{15}$ to the bis(difluoroboron) scaffold. In addition, a (2-hydroxyphenyl)ethylidene-substituted BOPHY probe for $\mathrm{Cu}^{2+}$ has been recently synthesized by $\mathrm{Li}$ and coworkers ${ }^{16}$, which is able of first coordinating the target, and next, through the hydrolysis of the $\mathrm{C}=\mathrm{N}$ bond, generating a formyl-substituted fluorescent BOPHY.

To the best of our knowledge, there has been only one previous theoretical study concerning BOPHYs ${ }^{17}$. This work concerned the simulation of one- and two-photon absorption bands of bis $\left(\mathrm{BF}_{2}\right)$ complexes containing naphthyridine derivatives and it revealed the good performance of the State Specific Polarizable Continuum Model (SS-PCM) approach in the 
estimation of the inhomogeneous broadening of the spectra. This initial study represents a valuable foundation for $a b$ initio calculations of the optical properties involving BOPHY molecules in solution. In the present contribution, we intend to study the optical properties of an extended set of BOPHY derivatives, using a Time-Dependent Density Functional Theory (TDDFT) protocol combined with a scaled opposite spin-configuration interaction singles with a double correction [SOS-CIS(D)] approach. In the first stage, we focus on the $0-0$ energies $\left(\mathrm{E}^{0-0}\right)$ calculations, given the interest of this parameter that allows physically well-grounded theory/experiment comparisons. $\mathrm{E}^{0-0}$ can be defined as the difference of the excited-state (ES) and ground-state (GS) energies taken at their respective optimal geometries and corrected for the difference of zero-point vibrational effects between the two states. $E^{0-0}$ can be accessed computationally and compared to the experimental absorption and fluorescence crossing point (AFCP, see Computational Details) ${ }^{18}$. Second, we simulate the vibrationally resolved spectra within the harmonic approximation on the basis of vibrational signatures. In this context, we note that vibronic TD-DFT calculations have been previously successfully employed to obtain the band shapes of (aza-) BODIPYs ${ }^{19}$, BODIPY-cyanines ${ }^{20}$ and Ladder-type- $\pi$-conjugated dyes ${ }^{21}$. Finally, we rationalize the effect of different lateral groups in the absorption/emission properties of the studied BOPHYs. To this end, we study electronic density difference (EDD) plots and charge transfer (CT) parameters. This work aims at providing some valuable guidelines to experimental teams working on the synthesis of BOPHY structures with improved optical properties. 


\section{COMPUTATIONAL DETAILS}

All DFT/TD-DFT calculations were carried out with the Gaussian09 software ${ }^{22}$, tightening self-consistent field convergence thresholds $\left(10^{-10}\right.$ a.u.). For all these calculations the M06-2X $\mathrm{X}^{23}$ hybrid exchange-correlation functional has been used. This choice of the exchangecorrelation functional is dictated by our previous works on (aza-) BODIPYs ${ }^{19}$, BODIPYcyanines $^{20}$ and Ladder-type- $\pi$-conjugated dyes ${ }^{21}$, all boron containing systems. Indeed, while this functional provides blue-shifted transition energies compared to experiment -an error common to all functionals when used in an adiabatic TD-DFT context for cyanine-like derivatives ${ }^{24}$ - the obtained structural and vibrational parameters were found accurate. All GS and ES geometry optimizations were followed by vibrational frequency calculations, where the absence of imaginary frequencies demonstrates the stability of the minimum of the structures. Two atomic basis sets, namely, 6-31G(d) for geometry optimizations and frequency calculations, and 6$311+\mathrm{G}(2 \mathrm{~d}, \mathrm{p})$ for electronic transitions have been applied. In order to reproduce the solvation effects, the Polarizable Continuum Model (PCM) is employed, using dichloromethane as solvent as in the experiments. In the PCM model, the solvent is described as a polarizable continuum medium characterized by its dielectric permittivity $\varepsilon$ and other macroscopic parameters. The interaction with the solute is modeled through a charge distribution on the cavity ${ }^{25,26}$. The equilibrium limit is considered for geometry optimizations, whereas the non-equilibrium limit is applied for calculations of both absorption and emission energies. In the framework of excitedstate calculations with $\mathrm{PCM}^{27}$, there are several approaches to account for the density reorganization, either using the transition matrix in the so-called Linear Response (LR) approach $^{28,29}$, or the one-particle density matrix using the corrected Linear Response (cLR) ${ }^{30}$ or State-Specific (SS) ${ }^{31}$ schemes. A combined protocol including TD-DFT and SOS-CIS(D) has 
been used to reduce the overestimation obtained in the TD-DFT excitation energies. Grimme and coworkers evaluated the performance of CIS(D) for the prediction of 0-0 excitation energies of large organic molecules, and they concluded that it represents a reliable and efficient approach given its size-consistency and relatively low computational cost ${ }^{32-34}$. However, it is clear that the range of CIS(D) application is limited by some requirements including a good description of the ground- and excited-states by the HF determinant and the CIS reference function, respectively $^{35,36}$. In addition, it was reported that CIS(D) sometimes yields significant blue shifts of more than several tenths of an $\mathrm{eV}$, due to near-degeneracy effects ${ }^{37}$. Moreover, the CIS(D) method and its variations are in practice much less efficient than TD-DFT for determining ES vibrational structures and geometries, and for this reason the CIS(D) calculations are often coupled to a TD-DFT protocol ${ }^{19}$ in which the former is used to determine transition energies and the latter the structural parameters. The SOS-CIS(D) variation used herein involves a single scaling parameter that influences the opposite-spin direct term of CIS(D) starting from the oneparameter scaled opposite spin MP2 ground-state ${ }^{38}$. This method has been shown to yield very satisfying results for several classes of dyes ${ }^{19,20,21,39-41}$. The SOS-CIS(D) calculations have been computed with the Q-Chem package ${ }^{42}$ with the $6-311+\mathrm{G}(2 \mathrm{~d}, \mathrm{p})$ basis set and by applying the resolution of the identity scheme, with a triple- $\zeta$ auxiliary basis set. Both CIS(D) and SOSCIS(D) partially account for the impact double excitations that are known to be important in boron difluoride dyes ${ }^{24}$. The AFCP energies were calculated with a recently designed protocol ${ }^{43-}$ ${ }^{45}$, that is briefly summarized below.

In the gas phase, a (idealized) vertical absorption $\left(E^{\text {vert-abs }}\right)$ can simply be defined as the difference between the ES and GS energies ( $E^{\mathrm{ES}}$ and $E^{\mathrm{GS}}$, respectively) at the optimal groundstate geometry $\left(R^{\mathrm{GS}}\right)$, 


$$
E^{\mathrm{vert}-\mathrm{abs}}=E^{\mathrm{ES}}\left(R^{\mathrm{GS}}\right)-E^{\mathrm{GS}}\left(R^{\mathrm{GS}}\right)
$$

whereas the vertical fluorescence $\left(E^{\text {vert-fluo }}\right)$ can be calculated in a similar manner, but considering the excited-state geometry.

$$
E^{\text {vert-fluo }}=E^{\mathrm{ES}}\left(R^{\mathrm{ES}}\right)-E^{\mathrm{GS}}\left(R^{\mathrm{ES}}\right)
$$

The adiabatic energy $\left(E^{\text {adia }}\right)$ is the difference of total electronic energies computed for the ES and GS in their corresponding optimal geometries:

$$
E^{\text {adia }}=E^{\mathrm{ES}}\left(R^{\mathrm{ES}}\right)-E^{\mathrm{GS}}\left(R^{\mathrm{GS}}\right)
$$

It can be calculated alternatively by combining vertical transition energies with the geometrical reorganization energies $\left(E^{\text {reorg }}\right)$, that measure the energy difference determined for a given single electronic state when considering the optimal GS and ES geometries:

$$
E^{\text {adia }}=\frac{1}{2}\left[E^{\text {vert-fluo }}+E^{\text {vert-abs }}\right]+\frac{1}{2}\left[E^{\text {reorg-GS }}-E^{\text {reorg-ES }}\right]
$$

In this latter equation the first term is dominant and we can make the following approximation:

$$
E^{\text {adia }} \simeq \frac{1}{2}\left[E^{\text {vert-fluo }}+E^{\text {vert-abs }}\right]
$$

At the right-hand side of this last equation, we can observe the average between the vertical absorption and emission. Next, we determine the difference of zero-point vibrational energy $\left(E^{\mathrm{ZPVE}}\right)$ between the ES and GS,

$$
\Delta E^{\mathrm{ZPVE}}=E^{\mathrm{ZPVE}}\left(R^{\mathrm{ES}}\right)-E^{\mathrm{ZPVE}}\left(R^{\mathrm{GS}}\right)
$$

to reach the 0-0 energy $\left(E^{0-0}\right)$ we add $E^{\text {adia }}$ and $\Delta E^{\mathrm{ZPVE}}$, 


$$
E^{0-0}=E^{\text {adia }}+\Delta E^{\mathrm{ZPVE}}
$$

$E^{0-0}$ can be compared to the experimentally measured absorption-fluorescence crossing point (AFCP), and it therefore offers a solid basis for theory-experiment comparisons.

For a representative system, the vibrationally resolved spectrum within the harmonic approximation was computed by using the FCclasses program on the basis of TD-DFT vibrational signatures $^{46-49}$. The reported spectrum was simulated by using a convoluting Gaussian function presenting a half width at half-maximum (HWHM) that was adjusted to allow direct comparison with the experimental spectrum (HWHM was set to $0.085 \mathrm{eV}$ and $0.055 \mathrm{eV}$ for absorption and fluorescence, respectively). A maximal number of 25 overtones for each mode and 20 combination bands for each pair of modes were included in the calculation. The maximum number of integrals to be computed for each class was set to $10^{6}$, which provided optimal spectra.

To visualize the electronic density redistribution and the possible intramolecular charge transfer we represented the difference between the total densities of the excited- and groundstates (EDD) as provided by TD-DFT,

$$
\Delta \rho(r)=\rho^{\mathrm{ES}}(r)-\rho^{\mathrm{GS}}(r)
$$

All EDD maps and HOMO-LUMO isosurfaces have been plotted with the Chemcraft $\operatorname{code}^{50}$, considering a contour threshold of 0.001 a.u. for the former and 0.03 a.u. for the latter. Additionally, the amount of CT ( $\mathrm{q}_{\mathrm{CT}}$ in $\left.|\mathrm{e}|\right)$, CT distance $\left(\mathrm{d}_{\mathrm{CT}}\right.$ in $\AA$ ), and CT dipole ( $\mu_{\mathrm{CT}}$ in Debye) have been calculated according to the methodology proposed by Le Bahers and coworkers ${ }^{51,52}$. 


\section{RESULTS AND DISCUSSION}

\section{Statistical Analysis}

In previous works dealing with the modeling of spectroscopic properties of BODIPY derivatives, the PCM-TD-DFT protocol enabled to obtain theoretical results in good qualitative agreement with experimental data, but generally too large on the absolute energy scale ${ }^{18-21}$. Here, we have tested two models for transition energies, TD-DFT and SOS-CIS(D), in both gas phase or combined with three solvent approaches (LR, cLR, and SS) in order to check which approach is the most effective in reproducing the experimental AFCP values for the thirteen BOPHY dyes represented in Scheme 2. The experimental AFCP energies and their theoretical counterparts are listed in Table 1, whereas the results of a statistical analysis providing the mean absolute error (MAE), mean square error (MSE), standard deviation (SD), maximal positive and negative deviations $[\operatorname{Max}(+)$ and $\operatorname{Max}(-)]$, as well as theory/experiment linear determination coefficients $\left(\mathrm{R}^{2}\right)$ can be found in Table 2. First of all, let us compare the experimental AFCP values, and the 0-0 energies obtained by TD-DFT and SOS-CIS(D) in gas phase (Figure 1). The obtained results evidence that without considering solvent effects the AFCP trend is rather well reproduced by TD-DFT with a $\mathrm{R}^{2}$ of 0.935 but at a cost of a very large MAE $(0.426 \mathrm{eV})$ and a sizeable MSE $(0.185 \mathrm{eV})$. Conversely, the gas-phase SOS-CIS(D) calculations provide much smaller average errors (MAE of $0.094 \mathrm{eV}$ and MSE of $0.013 \mathrm{eV}$ ), but do not enable to accurately reproduce the observed trend $\left(\mathrm{R}^{2}=0.869\right)$. This is mainly due to an overestimation of the $0-0$ values for those BOPHYs that absorb light at small energies (5, 6 and 7). Similar deviations in the SOS-CIS(D) results have been evidenced by Goerigk and Grimme for a panel of twelve chromophores of different nature ${ }^{32}$ 
Next, we carried out the same study by TD-DFT methodologies incorporating the solvent effects via the state specific (SS-TD-DFT), linear response (LR-TD-DFT) and corrected linear response (cLR-TD-DFT) schemes (Figure 2). An improvement in terms of correlation is observed. The cLR-TD-DFT approach leads us to the highest $\mathrm{R}^{2}$ value of 0.968 , whereas SS and LR provide values of 0.965 and 0.955 , respectively. As in the gas phase, the incorporation of SOS-CIS(D) deteriorates these coefficients, but only slightly here, with values of 0.941 for LR, 0.950 for cLR and 0.936 for SS. Again, the MAE and MSE values clearly show the positive impact of the inclusion of the SOS-CIS(D) corrections in terms of accuracy. Indeed, compared to TD-DFT the MAE (MSE) values decrease from $0.221 \mathrm{eV}(0.052 \mathrm{eV})$ to $0.199 \mathrm{eV}(0.046 \mathrm{eV})$ for $\mathrm{LR}$, from $0.431 \mathrm{eV}(0.188)$ to $0.061 \mathrm{eV}(0.005 \mathrm{eV})$ with cLR, and from $0.521 \mathrm{eV}(0.277 \mathrm{eV})$ to $0.104 \mathrm{eV}(0.014 \mathrm{eV})$ with SS. In Figure 2, one clearly sees that TD-DFT overestimates the experimental AFCP energies, irrespective of the selected solvent models, which was, as stated above, expected from previous simulations of fluoroborates ${ }^{19,21}$. SS-SOS-CIS(D) also slightly overshoots the experimental data, whereas we found a significant underestimation with LR-SOSCIS(D). Overall, the most accurate approach is cLR-SOS-CIS(D) that delivers only a trifling average underestimation compared to the experimental results. It is also interesting to note that to obtain both accurate absolute values and high correlation, one needs the inclusion of both solvent effects and the impact of "double excitation" in the calculation, highlighting the challenge of theoretically modeling BOPHY with high accuracy. 


\section{Analysis of the band topology}

Globally, the shapes of the absorption and emission bands of BOPHYs are rather independent of their substitution patterns, suggesting that vibronic couplings can be attributed to the BOPHY core rather than the auxochromes. Consequently, we investigated the vibronic couplings of molecule $\mathbf{1}$ as a representative example, by applying the FC approximation, and the spectra are presented in Figure 3. These results are perfectly converged with FC factors exceeding 0.95 for both absorption and emission. It is rather obvious that theory reproduces very nicely both the position and the band shapes compared to experiment of Ref. 11, the only slight difference being the relative heights of the two maxima in the absorption spectrum that are inverted by the model. Unlike the BODIPY dyes, where the emission profile is the mirror image of the absorption one, a clear difference exists between them for BOPHY, and this is well reproduced by our calculations. Interestingly the computed absolute molar extinction coefficient, $\varepsilon$, that is $4.110^{4} \mathrm{M}^{-1} \mathrm{~cm}^{-1}$, has the correct order of magnitude compared to the experiment, 7.0 $10^{4} \mathrm{M}^{-1} \mathrm{~cm}^{-1}$, which also corroborates the quality of the FC model in the present case. Additionally, we have analyzed the transitions playing a key role in the band shapes. For the absorption spectrum, one mode is highly relevant: an excited-state vibration at $1533 \mathrm{~cm}^{-1}$, that is responsible for both, the second maximum (one quantum) and the significant broadening at higher energies (two quanta). This mode is mainly related to the intense stretching of the central $\mathrm{N}-\mathrm{N}$ bond. This explains why the typical band shape of BODIPY is not recovered in BOPHY: the new N-N linkage plays a key role in the vibronic couplings, and hence, in the band shape. For the emission, the marked shoulder is principally due to a ground-state vibration at $1397 \mathrm{~cm}^{-1}$ (see Supporting Information). This mode involves the central N-N bond as well, but also includes a marked wagging of the hydrogen atoms. The different natures of the coupling modes 
for absorption and emission are consistent with the unsymmetrical band topologies measured experimentally.

\section{Structure-Property Relationships}

The BOPHY framework is characterized by two fused $\mathrm{BF}_{2}$-containing six-membered rings in the center and two five-membered pyrrole moieties at the periphery. Whereas the pyrrole and $\mathrm{BF}_{2}$-containing units are coplanar, there exists a central dihedral angle of ca. $28.5^{\circ}$ in all

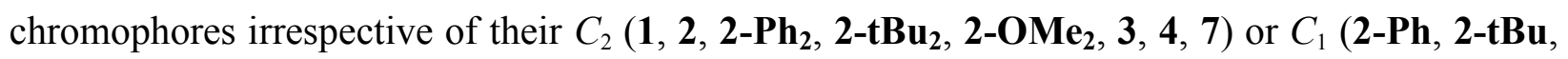
2-OMe, 5, 6) symmetry (see Table 3). It seems that this dihedral angle remains unaltered despite the addition of different substituents, in contrast with the BODIPY fluorophores in which the incorporation of lateral substituents usually favors structural planarity ${ }^{53}$. In all cases, the central dihedral angle is reduced in the excited-state, denoting a greater $\pi$-conjugation in $S_{l}$ (see Table 3). Additionally, a reduction of the central N-N bond distance occurs compared to the groundstate, whereas the B-N distances do not present significant variations.

The HOMO-LUMO plots in Figure 4 for six representative BOPHY systems reveal that the HOMO is mainly spread over the nitrogen atoms and peripheral rings, with the exception of compounds 5 and 7 in which dimethylaminophenyl and methoxyphenyl groups connected through vinyl linkers also participate significantly. In contrast, the LUMO is distributed over the pyrrole units and the meso- carbon atoms, i.e., those adjacent to the central N-N bond. Recently, Nemykin and coworkers attempted to introduce a cyano substituent to this meso-position, but the dramatic reduction of the nitrogen atom basicity resulted in a partial extrusion of the BOPHY core $^{54}$ 
The incorporation of methyl groups in the $\alpha$ - and $\beta$-positions of the pyrrole units (Scheme 1) of 1 generates the tetra-methyl substituted compound 2 and causes a redshift of $+20 \mathrm{~nm}$ ($0.132 \mathrm{eV})$ in absorption, and $+20 \mathrm{~nm}(-0.110 \mathrm{eV})$ in emission. The subsequent addition of ethyl groups to the $\beta$-positions leading to 3 induces additional bathochromic shifts of $+34 \mathrm{~nm}(-0.199$ $\mathrm{eV})$ and $+12 \mathrm{~nm}(-0.062 \mathrm{eV})$ in the absorption and emission, respectively. Similar red-shift magnitudes are obtained when alkyl groups are added to the $\beta$-positions of BODIPYs ${ }^{8}$.

The inclusion of a phenylacetylene group in $\mathbf{2}$ leads to compound $\mathbf{2}-\mathbf{P h}$ which yields absorption and emission redshifts of $+12 \mathrm{~nm}(-0.073 \mathrm{eV})$ and $+24 \mathrm{~nm}(-0.121 \mathrm{eV})$, respectively, evidencing the effective but rather limited electronic communication between the BOPHY moiety and the phenyl group through the ethynyl linker. However, the electronic density difference (EDD) maps (Figure 5) reveal that this linker could actually act as a secondary electron donor group, which is consistent with the absorption [+9nm $(-0.055 \mathrm{eV})]$ and emission $[+14 \mathrm{~nm}(-0.072 \mathrm{eV})]$ red-shifts of similar magnitude observed in 2-tBu. In this context, let us recall that incorporating tert-butyl substituents advantageously limits $\pi$ - $\pi$ aggregation in solidstate, which allows to avoid the so-called solid-state emission quenching ${ }^{55,56}$.

The electron-donor character of the methoxyphenyl group is capable to generate redshifts in aza-boron-dipyrromethene dyes, when this group is incorporated into $\alpha$-positions ${ }^{57}$. Analogously, in BOPHY dyes the influence of one methoxyphenyl attached to the pyrrole unit through an ethynyl linker (2-OMe) causes a bathochromic shift of $+35 \mathrm{~nm}(-0.204 \mathrm{eV})$ for the absorption and $\mathrm{a}+38 \mathrm{~nm}(-0.186 \mathrm{eV})$ shift for the fluorescence, with respect to 2 , more pronounced than those observed in $\mathbf{2}-\mathbf{P h}$ and $\mathbf{2 - t} \mathbf{B u}$. The large $\mathrm{q}_{\mathrm{CT}}$ value calculated for the $\mathbf{2}$ OMe system $(0.511|\mathrm{e}|)$ evidences a synergic effect caused by the ethynyl linker and the methoxyphenyl moiety. 
In 3-dimethylaminophenyl-8- $\mathrm{CF}_{3}-\mathrm{BODIPY}$, the addition of the strong electron-donor amino groups to the pyrrole units triggers an intramolecular charge transfer leading to bathochromic red-shifts greater than $+100 \mathrm{~nm}$ for both absorption $(-0.230 \mathrm{eV})$ and emission maxima $(-0.209 \mathrm{eV})^{58}$. In the BOPHY case, compounds 5 and $\mathbf{6}$-where the electron-donor substituent 4-dimethylaminophenyl is linked through a vinyl connector- present larger bathochromic displacements in comparison to their $\mathbf{2}$ and $\mathbf{3}$ analogues. Hence, $\mathbf{5}$ exhibits an absorption red-shift of $+113 \mathrm{~nm}(-0.567 \mathrm{eV})$ and an emission red-shift of $+182 \mathrm{~nm}(-0.698 \mathrm{eV})$ with respect to 2 , whereas 6 is red-shifted in $+79 \mathrm{~nm}(-0.368 \mathrm{eV})$ in absorption and $+173 \mathrm{~nm}(-$ $0.644 \mathrm{eV}$ ) in emission, with respect to 3 . Moreover, these two chromophores display the largest absorption and emission wavelengths of the panel, which can be explained by their pronounced donor-acceptor character, evidenced by high charge transfer dipole $\left(\mu_{\mathrm{CT}}\right)$ values of $10.3 \mathrm{D}(\mathbf{5})$ and $10.0 \mathrm{D}(\mathbf{6})$ (see Table 4). In addition, the greatest $\mathrm{q}_{\mathrm{CT}}$ values of our set of dyes are obtained with these unsymmetrical chromophores $(0.60|\mathrm{e}|$ for both $\mathbf{5}$ and $\mathbf{6})$. The EDD plots also reveal the strong variation experienced by the electronic densities of the donor lateral arms, demonstrating the efficient $\pi$-conjugation achieved by the vinyl linkers. In contrast, the presence of two methoxyphenyl groups in the $\alpha$-positions (7) decreases the dipole CT character of the molecule, resulting in a smaller emission maximum $(611 \mathrm{~nm})$. While the $\mu_{\mathrm{CT}}$ is not significant for the symmetric dye 7 , we notice that its $\mathrm{q}_{\mathrm{CT}}(0.516|\mathrm{e}|)$ is significantly smaller than those corresponding to $\mathbf{5}$ and $\mathbf{6}$. Another advantageous feature exhibited by $\mathbf{5}$ and $\mathbf{6}$ is their large Stokes shift of $2961 \mathrm{~cm}^{-1}$ and $3028 \mathrm{~cm}^{-1}$, respectively, probably preventing re-absorption of emitted photons that might lead to undesired background interferences ${ }^{59}$. At this point we can state that the very large absorption and emission maxima found for $\mathbf{5}$ and $\mathbf{6}$ chromophores, near 
the so-called first biological window ${ }^{60}$ and their large Stokes shifts, make them an interesting starting point for the design of novel bioluminescence agents.

Increasing $\pi$-electron conjugation of similar emissive organic chromophores normally results in enhanced fluorescent emission, as well as redshifts in excitation and emission wavelengths ${ }^{61-64}$. This strategy is applied in system 4, a naphthyridin derivative ${ }^{65}$ where the $\pi$ conjugation is extended by fusing the pyrrole units with other aromatic rings. We found that absorption maximum is red-shifted in $+54 \mathrm{~nm}(-0.303 \mathrm{eV})$, and emission in $+95 \mathrm{~nm}(-0.419 \mathrm{eV})$, with respect to $\mathbf{2}$, as a result of the donor effect of the added rings (see EDD map). In the BODIPY case, by extending the central core, red-shifts of $+39 \mathrm{~nm}(-0.125 \mathrm{eV})$ and $+42 \mathrm{~nm}(-$ $0.130 \mathrm{eV}$ ) are found for absorption and emission, respectively ${ }^{63}$, while in the case of azaBODIPYs, smaller displacements of ca. $+16(-0.045 \mathrm{eV})$ are found for both absorption and emission $^{64}$ 


\section{CONCLUDING REMARKS}

We have investigated a large number of BOPHY dyes that present exciting phophysical signatures for applications in several fields. The 0-0 energies trend for the studied BOPHY systems is well reproduced by a protocol relying on TD-DFT for excitation energies and cLRPCM for solvent effects as evidenced by a $\mathrm{R}^{2}$ of 0.968 when comparisons with experiments are made. However, this scheme yields large values of both the MAE $(0.431 \mathrm{eV})$ and MSE $(0.188$ eV) compared to experiment. The subsequent incorporation of a SOS-CIS(D) correction of the transition energies considerably improves both the MAE $(0.061 \mathrm{eV})$ and MSE $(0.005 \mathrm{eV})$ values, at the cost of only a very slight deterioration of the determination coefficient (0.950). The cLRTD-DFT/SOS-CIS(D) mixed approach therefore allows to reproduce all key experimental features with high accuracy and is a method of choice to model and design BOPHY derivatives.

A fine tuning of the spectroscopic properties of the BOPHY dyes can be achieved by structural modifications involving the incorporation of different substituents to the pyrrole units. Remarkably, the incorporation of one dimethylaminophenyl group via a vinyl linker in the $\alpha$ position gives rise to two BOPHYs with particularly high absorption and emission maxima (5 and 6). We have rationalized these effects as resulting from a pronounced donor-acceptor behavior absent in the parent structures. These high absorption/emission maxima, along as the large Stokes shifts found for both systems, make them promising candidates for bioluminescence applications. It is therefore our hope that this work will stimulate the modeling of new BOPHY derivatives for biological applications. 
FIGURES

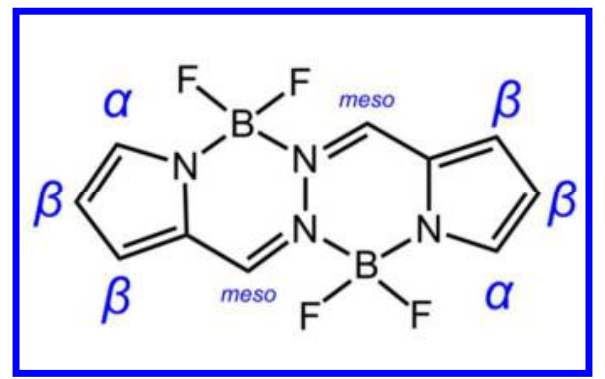

Scheme 1. Typical Structure of a BOPHY dye.

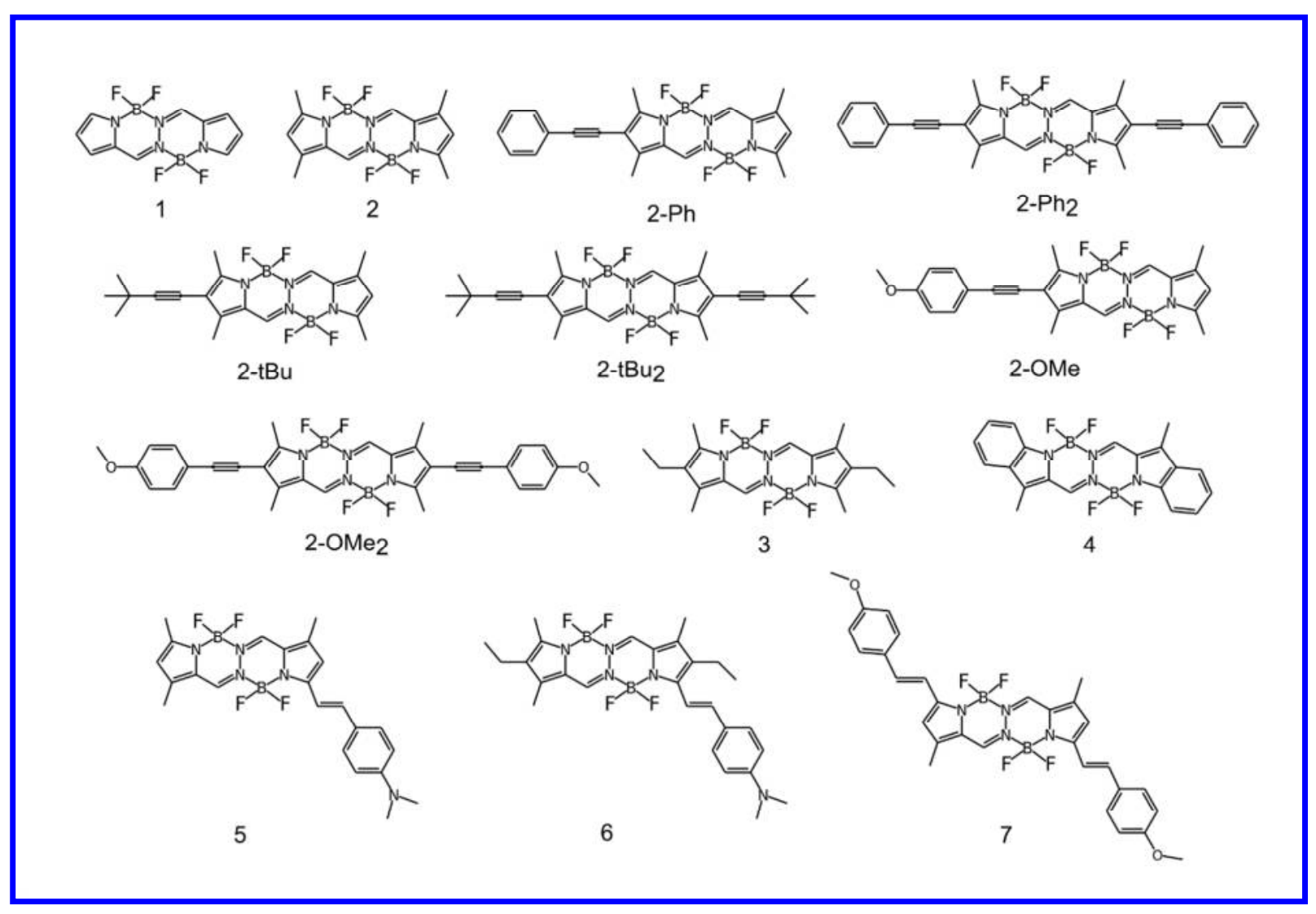

Scheme 2. Representation of the complete set of BOPHY systems studied in the present work. 


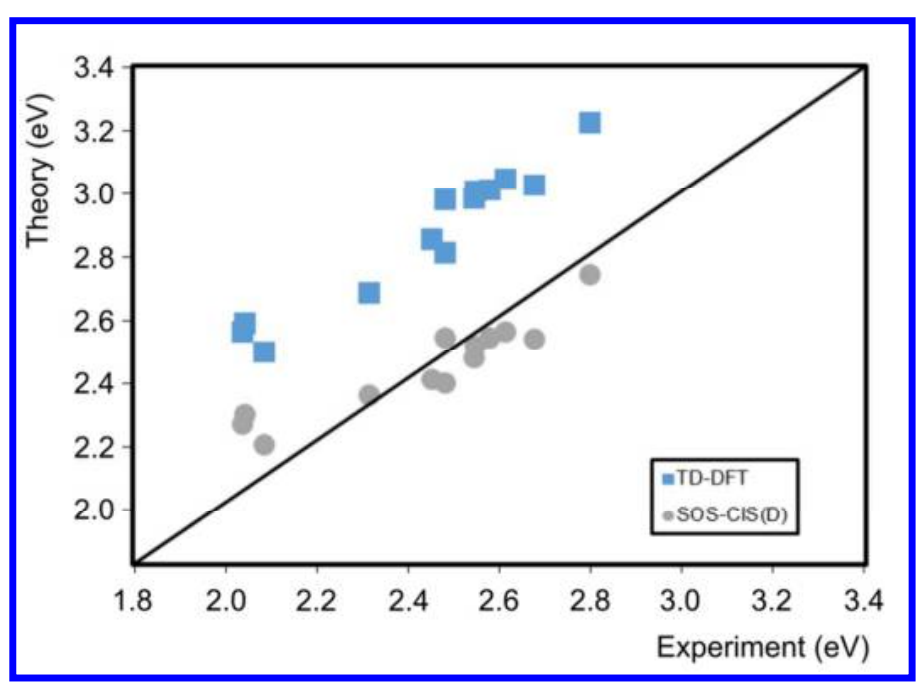

Figure 1. Comparison between theoretical values without considering solvent effects [TD-DFT and SOS-CIS(D)] and experimental AFCP values (eV). The central line indicates a perfect theory-experiment match.

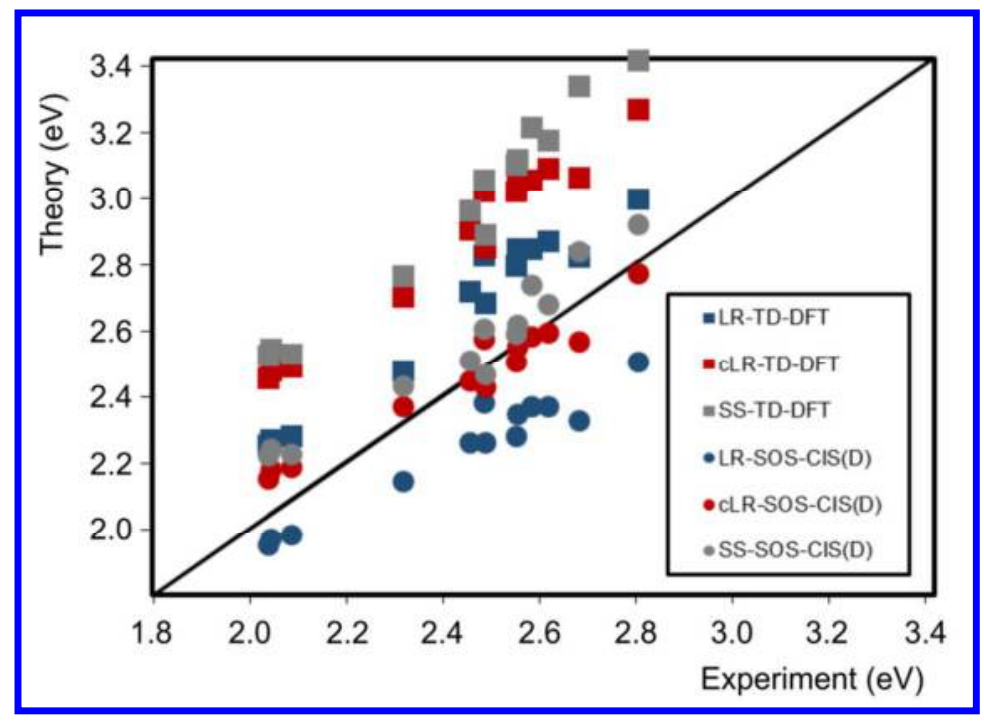

Figure 2. Comparison between theoretical (LR-TD-DFT, cLR-TD-DFT, SS-TD-DFT, LR-SOSCIS(D), cLR-SOS-CIS(D) and SS-SOS-CIS(D)) and experimental AFCP values (eV). All calculations are carried out considering dichloromethane as solvent. The central line indicates a perfect theory-experiment match. 


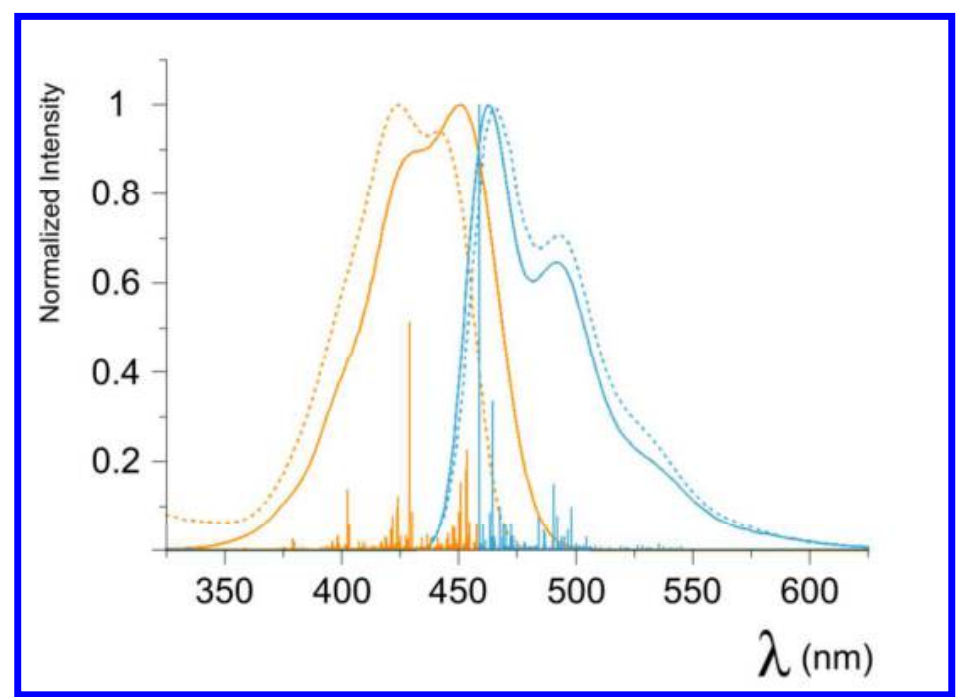

Figure 3. Comparisons between the experimental (dotted lined) and theoretical (full lines and sticks) normalized absorption (in orange) and emission (in blue) spectra of 1 , in dichloromethane solution. The experimental curve is adapted from Ref. 11.

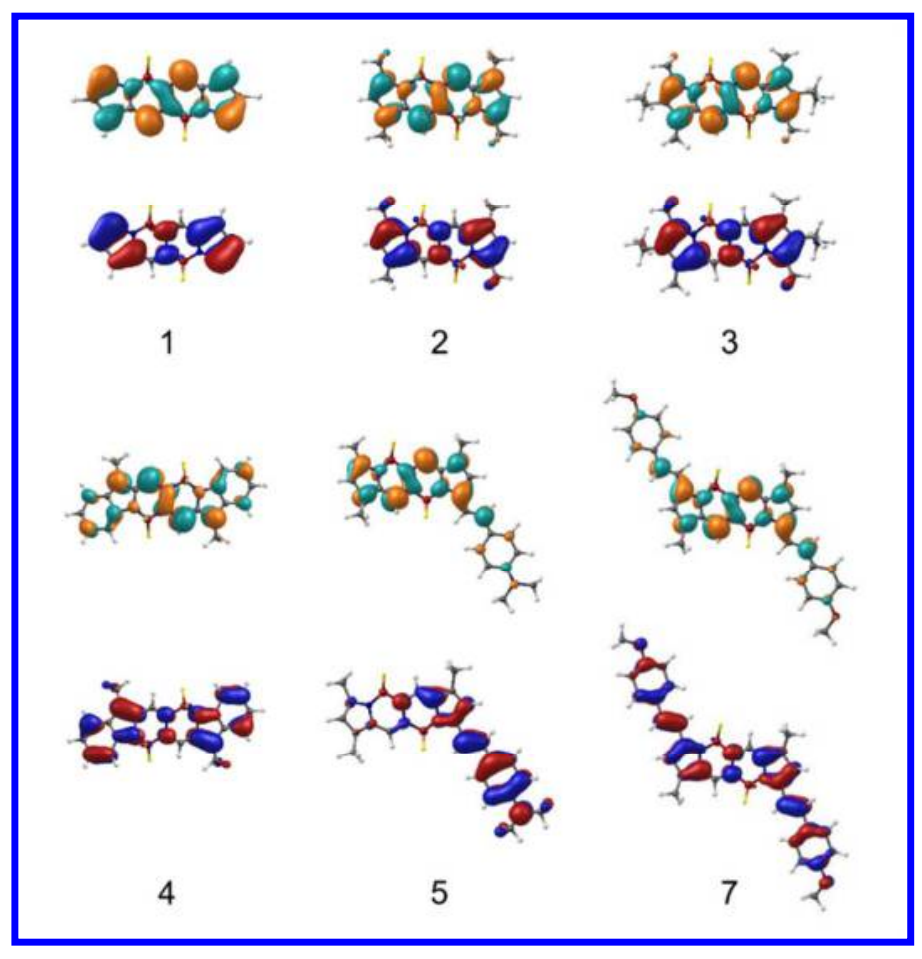

Figure 4. HOMO-LUMO plots for some selected chromophores. The HOMO is represented in red-blue, and the LUMO in turquoise-orange. A contour threshold of 0.03 a.u. has been applied. 


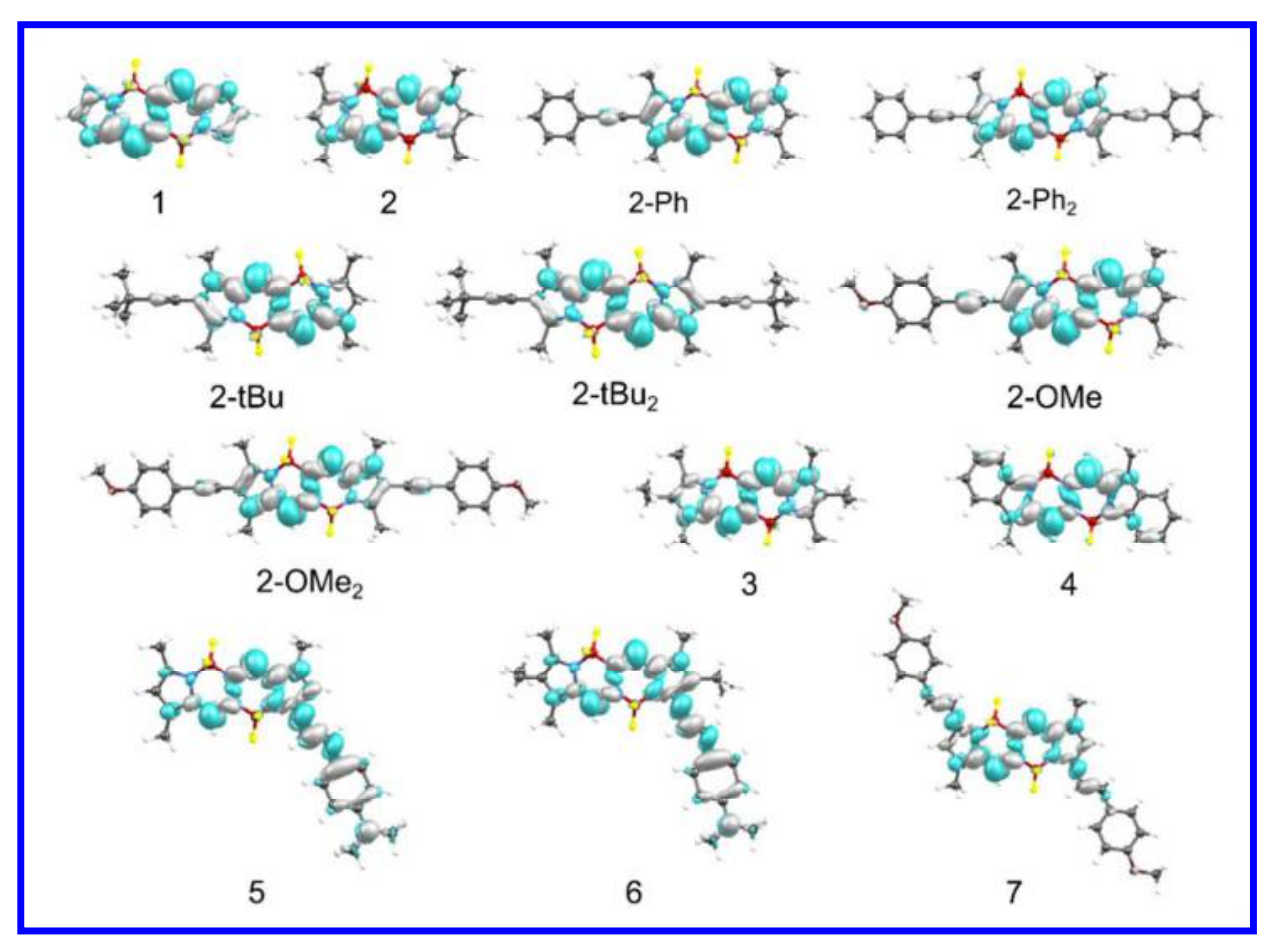

Figure 5. Electronic Density Difference plots for the studied systems. Turquoise and gray colors indicate density accumulation and depletion, respectively. A contour threshold of 0.001 a.u. has been applied. 


\section{TABLES}

Table 1. Experimental AFCP energies (eV) and their corresponding calculated values using various computational models. All calculations were carried out considering dichloromethane as solvent.

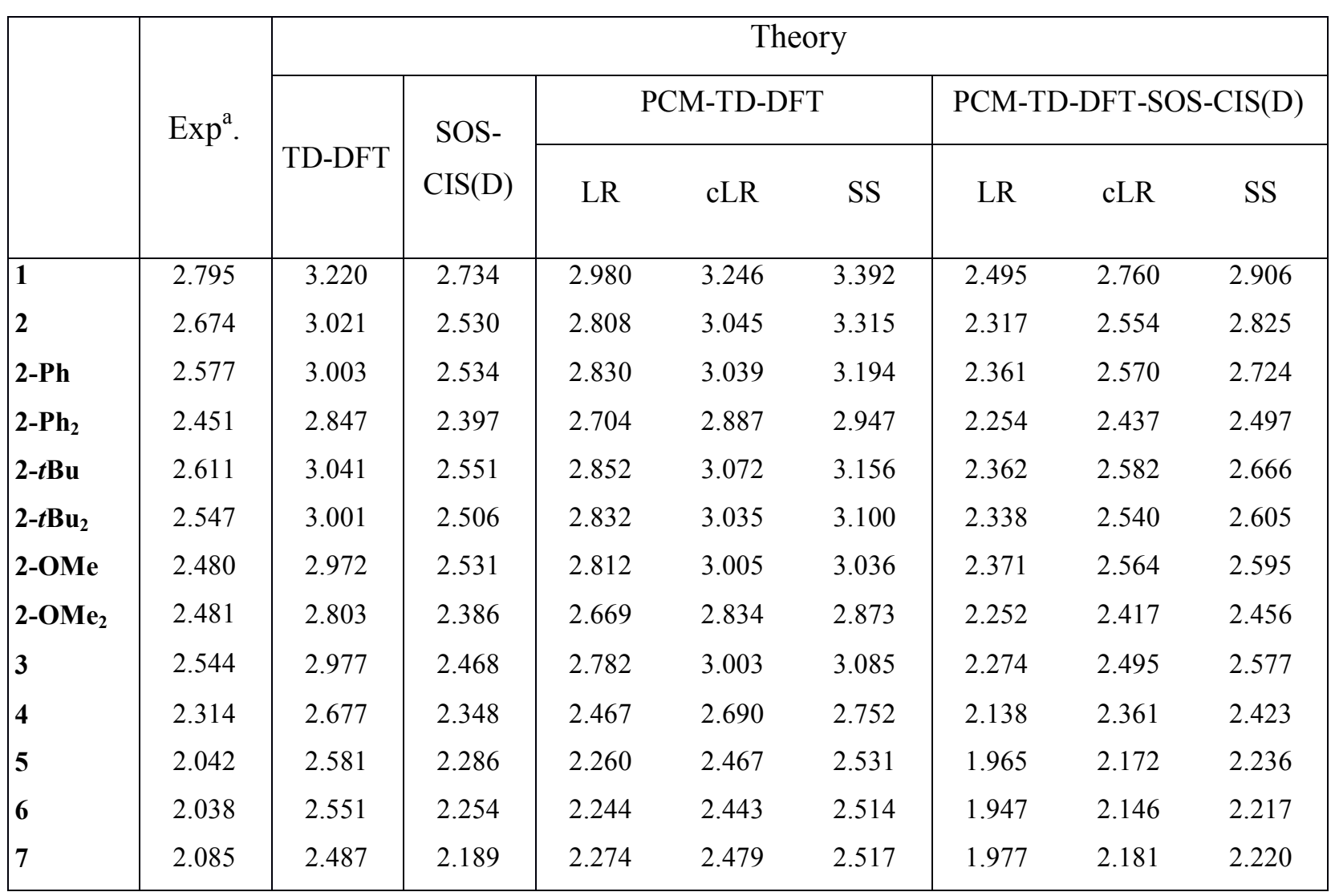

a Experimental data from references $11,12,14$ and 16 
Table 2. Statistical analysis obtained from the comparison of experimental and theoretical AFCP energies (Table 1): mean absolute error (MAE), mean square error (MSE), standard deviation (SD), maximal positive and negative deviations $[\operatorname{Max}(+)$ and $\operatorname{Max}(-)]$, and theory/experiment linear determination coefficients $\left(\mathrm{R}^{2}\right)$. All values except those for $\mathrm{R}^{2}$ are given in eV. All calculations except those concerning the first two lines [TD-DFT and SOS-CIS(D)] were carried out considering dichloromethane as solvent.

\begin{tabular}{|c|cccccc|}
\hline & MAE & MSE & SD & $\operatorname{Max}(+)$ & $\operatorname{Max}(-)$ & $\mathrm{R}^{2}$ \\
\hline TD-DFT & 0.426 & 0.185 & 0.224 & 0.538 & 0.322 & 0.935 \\
SOS-CIS(D) & 0.094 & 0.013 & 0.148 & 0.244 & -0.144 & 0.869 \\
& & & & & & \\
LR-TD-DFT & 0.221 & 0.052 & 0.255 & 0.332 & 0.133 & 0.955 \\
cLR-TD-DFT & 0.431 & 0.188 & 0.264 & 0.525 & 0.353 & 0.968 \\
SS-TD-DFT & 0.521 & 0.277 & 0.299 & 0.641 & 0.392 & 0.965 \\
& & & & & & \\
LR-SOS-CIS(D) & 0.199 & 0.046 & 0.175 & -0.077 & -0.357 & 0.941 \\
cLR-SOS-CIS(D) & 0.061 & 0.005 & 0.185 & 0.130 & -0.120 & 0.950 \\
SS-SOS-CIS(D) & 0.104 & 0.014 & 0.223 & 0.194 & -0.025 & 0.936 \\
\hline
\end{tabular}


Table 3. Selected ground-state and excited-state dihedral angles (degrees) and bond distances (Å) for the optimized structures of the studied BOPHYs

\begin{tabular}{|c|c|c|c|c|c|c|c|}
\hline Dihedral & 1 & 2 & 2-Ph & $2-P_{2}$ & $2-t \mathrm{Bu}$ & $2-t \mathrm{Bu}_{2}$ & 2-OMe \\
\hline GS & 29.1 & 28.3 & 28.6 & 28.9 & 28.6 & 28.7 & 28.7 \\
\hline $\mathrm{ES}$ & 23.1 & 21.9 & 22.6 & 23.0 & 22.3 & 22.6 & 22.9 \\
\hline \multicolumn{8}{|l|}{$\mathrm{N}-\mathrm{N}$} \\
\hline GS & 1.390 & 1.389 & 1.388 & 1.389 & 1.389 & 1.389 & 1.388 \\
\hline $\mathrm{ES}$ & 1.326 & 1.328 & 1.329 & 1.330 & 1.328 & 1.328 & 1.331 \\
\hline \multicolumn{8}{|l|}{$\mathrm{N}_{\text {(central) }}-\mathrm{B}$} \\
\hline GS & 1.602 & 1.596 & 1.593 & 1.317 & 1.317 & 1.318 & 1.593 \\
\hline $\mathrm{ES}$ & 1.601 & 1.607 & 1.605 & 1.605 & 1.606 & 1.357 & 1.603 \\
\hline Dihedral & $2-\mathrm{OMe}_{2}$ & 3 & 4 & 5 & 6 & 7 & \\
\hline GS & 28.8 & 27.9 & 28.4 & 28.6 & 28.3 & 29.0 & \\
\hline ES & 23.3 & 21.3 & 23.5 & 24.6 & 24.3 & 23.6 & \\
\hline \multicolumn{8}{|l|}{$\mathrm{N}-\mathrm{N}$} \\
\hline GS & 1.388 & 1.388 & 1.387 & 1.387 & 1.386 & 1.385 & \\
\hline ES & 1.331 & 1.328 & 1.336 & 1.350 & 1.349 & 1.337 & \\
\hline \multicolumn{8}{|l|}{$\mathrm{N}_{\text {(central) }}-\mathrm{B}$} \\
\hline GS & 1.597 & 1.595 & 1.615 & 1.592 & 1.593 & 1.593 & \\
\hline ES & 1.604 & 1.607 & 1.608 & 1.596 & 1.594 & 1.600 & \\
\hline
\end{tabular}


Table 4. Experimental absorption maxima (nm), emission maxima (nm), Stokes shift $\left(\mathrm{cm}^{-1}\right)$, and calculated CT charge $(|\mathrm{e}|)$, distance $(\AA)$, and dipole (Debye).

\begin{tabular}{|c|c|c|c|c|c|c|}
\hline & $\begin{array}{c}\text { Abs. max. } \\
(\mathrm{nm}) \\
\text { Exp }^{\mathrm{b}}\end{array}$ & $\begin{array}{c}\text { Em. max. } \\
(\mathrm{nm}) \\
\operatorname{Exp}^{\mathrm{b}}\end{array}$ & $\begin{array}{c}\text { Stokes } \\
\text { shift } \\
\left(\mathrm{cm}^{-1}\right) \\
\text { Exp }^{\mathrm{b}}\end{array}$ & $\begin{array}{c}\text { CT charge } \\
\left(\left|\mathrm{e}^{-}\right|\right)\end{array}$ & $\begin{array}{c}\mathrm{CT} \\
\text { distance } \\
(\AA)\end{array}$ & $\begin{array}{l}\text { CT dipole } \\
\text { (Debye) }\end{array}$ \\
\hline 1 & 424 & 465 & 2080 & 0.442 & - & - \\
\hline 2 & 444 & 485 & 1904 & 0.431 & - & - \\
\hline $2-\mathrm{Ph}$ & 456 & 509 & 2283 & 0.483 & 1.206 & 2.799 \\
\hline $2-P_{2}$ & 489 & 524 & 1366 & 0.518 & - & - \\
\hline $2-t \mathrm{Bu}$ & 453 & 499 & 2035 & 0.465 & 0.723 & 1.614 \\
\hline $2-t \mathrm{Bu}_{2}$ & 464 & 512 & 2020 & 0.490 & - & - \\
\hline 2-OMe & 479 & 523 & 1756 & 0.511 & 1.950 & 4.782 \\
\hline $2-\mathrm{OMe}_{2}$ & 468 & 536 & 2711 & 0.540 & - & - \\
\hline 3 & 478 & 497 & 800 & 0.446 & - & - \\
\hline 4 & 498 & 580 & 2839 & 0.458 & - & - \\
\hline 5 & 557 & 667 & 2961 & 0.603 & 3.563 & 10.317 \\
\hline 6 & 557 & 670 & 3028 & 0.602 & 3.468 & 10.033 \\
\hline 7 & 579 & 611 & 905 & 0.516 & - & - \\
\hline
\end{tabular}

b Experimental data from references 11, 12, 14 and 16 
ASSOCIATED CONTENT

Movies of the key vibrations (.avi files) are available free of charge at http://pubs.acs.org.

\section{AUTHOR INFORMATION}

\section{Corresponding Author}

Dr. Miguel Ponce-Vargas

Université Paris-Est, Laboratoire Modélisation et Simulation, Multi Echelle, MSME UMR 8208

CNRS, 5 bd Descartes, 77454 Marne-la-Vallée, France.

e-mail : miguel.poncevargas@u-pem.fr

\section{ACKNOWLEDGMENT}

M.P.-V. thanks the ANR (Project ANR-14-CE05-0035-02) for his postdoctoral grant. C. A. is indebted to the ANR for supporting her $\mathrm{PhD}$ in the framework of the EMA program. D. J. acknowledges the European Research Council (ERC) and the Région des Pays de la Loire for financial support in the framework of a Starting Grant (MARCHES-278845) and LumoMat Project, respectively. This research used resources of the Centre de Calcul Intensif des Pays de Loire (CCIPL), a local Troy cluster, and the CINES. 


\section{REFERENCES}

[1] Qu, X. Seeing the Unseen: Advances in Bioimaging and Biosensors. J. Mater. Chem. B 2016, 4, 4500-4501.

[2] Erathodiyil, N.; Ying, J. Y. Functionalization of Inorganic Nanoparticles for Bioimaging Applications Acc.Chem.Res. 2011, 44, 925-935.

[3] Ozawa, T.; Yoshimura, H.; Kim, S.-B. Advances in Fluorescence and Bioluminescence Imaging. Anal. Chem. 2013, 85, 590-609.

[4] Wu, X.; Zhu, W. Stability Enhancement of Fluorophores for Lighting up Practical Application in Bioimaging. Chem.Soc.Rev. 2015, 44, 4179-4184.

[5] Hamilton, G. R. C.; Sahoo, S. K.; Kamila, S.; Singh, N.; Kaur, N.; Hyland, B. W.; Callan, J. F. Optical Probes for the Detection of Protons, and Alkali and Alkaline Earth Metal Cations. Chem.Soc.Rev. 2015, 44, 4415-4432.

[6] Horváth, P.; Šebej, P.; Šolomek, T.; Klán, P. Small-Molecule Fluorophores with Large Stokes Shifts: 9-Iminopyronin Analogues as Clickable Tags. L. Org Chem. 2015, 80, 1299-1311.

[7] Yang, L.; Liu, Y.; Ma, C.; Liu, W.; Li, Y.; Li, L. Naphthalene-fused BODIPY with Large Stokes Shift as Saturated-red Fluorescent Dye for Living Cell Imaging. Dyes and Pigments, 2015, 122, 1-5.

[8] Loudet, A.; Burgess, K. BODIPY Dyes and Their Derivatives: Syntheses and Spectroscopic Properties. Chem.Rev. 2007, 107, 4891-4932. 
[9] Ulrich, G.; Ziessel, R.; Harriman, A. The Chemistry of Fluorescent Bodipy Dyes: Versatility Unsurpassed. Angew. Chem. Int. Ed. 2008, 47, 1184, 1201.

[10] Zhou, L.; Xu, D.; Gao, H.; Zhang, C.; Ni, F.; Zhao, W.; Cheng, D.; Liu, X.; Han, A. $\beta$ Furan-Fused bis(Difluoroboron)-1,2-bis((1H-pyrrol-2-yl)methylene)hydrazine Fluorescent Dyes in the Visible Deep-Red Region. L. Org. Chem. 2016, 81, 7439-7447.

[11] Tamgho, I.-S.; Hasheminasab, A.; Engle, J. T.; Nemykin, V. N.; Ziegler, C. J. A New Highly Fluorescent and Symmetric Pyrrole-BF 2 Chromophore: BOPHY. J. Am. Chem. Soc. 2014, 136, 5623-5626.

[12] Yu, C.; Jiao, L.; Zhang, P.; Feng, Z.; Cheng, C.; Wei, Y.; Mu, X.; Hao, E. Highly Fluorescent $\mathrm{BF}_{2}$ Complexes of Hydrazine-Schiff Base Linked Bispyrrole. Org. Lett. 2014, 16, 3048-3051.

[13] Majumdar, P.; Yuan, X.; Li, S.; Le Guennic, B.; Ma, J.; Zhang, C.; Jacquemin, D.; Zhao, J. Cyclometalated Ir(III) Complexes with Styryl-BODIPY Ligands showing Near IR Absorption/Emission: Preparation, Study of Photophysical Properties and Applications as Photodynamic/Luminescence Imaging Materials. L.Mater.Chem.B 2014, 2, 2838-2854.

[14] Huaulmé, Q.; Mirloup, A.; Retailleau, P.; Ziessel, R. Synthesis of Highly Functionalized BOPHY Chromophores Displaying Large Stokes Shifts. Org Lett. 2015, 17, 2246-2249.

[15] Rhoda, H. M.; Chanawanno, K.; King, A. J.; Zatsikha, Y. V.; Ziegler, C. J.; Nemykin, V. N. Unusually Strong Long-Distance Metal-Metal Coupling in Bis(ferrocene)-Containing BOPHY: An Introduction to Organometallic BOPHYs. Chem. Eur. J. 2015, 21, 18043-18046. 
[16] Li, Y.; Zhou, H.; Yin, S.; Jiang, H.; Niu, N.; Huang, H.; Shahzad, S. A.; Yu, C. A BOPHY Probe for the Fluorescence Turn-On Detection of $\mathrm{Cu}^{2+}$. Sens.Actuators B-Chem 2016, 235, 3338.

[17] Zaleśny, R.; Murugan, N. A.; Tian, G.; Medved', M.; Ågren, H. First-Principles Simulations of One- and Two-Photon Absorption Band Shapes of the $\operatorname{Bis}\left(\mathrm{BF}_{2}\right)$ Core Complex. J. Phys. Chem.B 2016, 120, 2323-2332.

[18] Charaf-Eddin, A.; Le Guennic, B.; Jacquemin, D. Optical Signatures of Borico Dyes: A TDDFT Analysis. Theor. Chem.Acc. 2014, 133, 1456.

[19] Chibani, S.; Laurent, A.; Le Guennic, B.; Jacquemin, D. Improving the Accuracy of Excited-State Simulations of BODIPY and Aza-BODIPY Dyes with a Joint SOS-CIS(D) and TD-DFT Approach. J.Chem. Theorv Comput. 2014, 10, 4574-4582.

[20] Charaf-Eddin, A.; Le Guennic, B.; Jacquemin, D. Excited-states of BODIPY-cyanines: ultimate TD-DFT challenges? RSC Adv. 2014, 4, 49449-49456.

[21] Chibani, S.; Laurent, A. D.; Le Guennic, B.; Jacquemin, D. Excited States of Ladder-Type $\pi$-Conjugated Dyes with a Joint SOS-CIS(D) and PCM-TD-DFT Approach. J. Phys. Chem. A 2015, 119, 5417, 5425 .

[22] Gaussian 09. Revision D. 01. Frisch, M. J. et al. Gaussian Inc., Wallingford CT, 2009.

[23] Zhao, Y.; Truhlar, D. G. The M062 Suite of Density Functionals for Main Group Thermochemistry, Thermochemical Kinetics, Non-covalent Interactions, Excited States, and 
Transition Elements: Two New Functionals and Systematic Testing of Four M062-Class Functionals and 12 Other Functionals. Theor. Chem. Acc. 2008, 120, 215-241.

[24] Le Guennic, B.; Jacquemin, D. Taking Up the Cyanine Challenge with Quantum Tools. Acc. Chem.Res. 2015, 48, 530-537.

[25] Tomasi, J.; Mennucci, B.; Cammi, R. Quantum Mechanical Continuum Solvation Models. Chem.Rev. 2005, 105, 2999-3093.

[26] Mennucci, B.; Tomasi, J.; Cammi, R.; Cheeseman, J. R.; Frisch, M. J.; Devlin, F. J.; Gabriel, S.; Stephens, P. J. Polarizable Continuum Model (PCM) Calculations of Solvent Effects on Optical Rotations of Chiral Molecules. J.Phvs. Chem.A 2002, 106, 6102-6113.

[27] Curutchet, C.; Muñoz-Losa A.; Monti, S.; Kongsted, J.; Scholes, G. D.; Mennucci, B. Electronic Energy Transfer in Condesed Phase Studied by a Polarizable QM/MM Model. J. Chem. Theorv Comput. 2009, 5, 1838-1848.

[28] Cammi, R.; Mennucci, B. Linear Response Theory for the Polarizable Continuum Model. J. Chem. Phys. 1999, 110, 9877-9886.

[29] Cossi, M.; Barone, V. Time-Dependent Density Functional Theory for Molecules in Liquid Solutions. L.Chem. Phvs. 2001, 115, 4708-4717.

[30] Caricato, M. A Corrected-Linear Response Formalism for the Calculation of Electronic Excitation Energies of Solvated Molecules with the CCSD-PCM Method. Comput. Theor. Chem. 2014, 1040-1041, 99-105. 
[31] Improta, R.; Scalmani, G.; Frisch, M. J.; Barone, V. Toward Effective and Reliable Fluorescence Energies in Solution by a New State Specific Polarizable Continuum Model Time Dependent Density Functional Theory Approach. LChem. Phvs. 2007, 127, 074504.

[32] Goerigk, L.; Moellmann, J.; Grimme, S. Computation of Accurate Excitation Energies for Large Organic Molecules with Double-Hybrid Density Functionals. Phys. Chem. Chem. Phys. 2009, 11, 4611-4620.

[33] Goerigk, L.; Grimme, S. Assessment of TD-DFT methods and of various spin scaled CIS(D) and CC2 versions for the treatment of low-lying valence excitations of large organic dyes. L.Chem. Phvs. 2010, 132, 184103.

[34] Grimme, S.; Goerigk, L.; Fink, R. F. Spin-Component-Scaled Electron Correlation Methods. WIREs Comput.Mol.Sci. 2012, 2, 886-906.

[35] Jacquemin, D.; Duchemin, I. Blase, X. 0-0 Energies Using Hybrid Schemes : Benchmarks of TD-DFT, CIS(D), ADC(2), CC2, and BSE/GW formalisms for 80 Real-Life Compounds. $J$. Chem. Theory, Comput. 2015, 11, 5340-5359.

[36] Moore II, B.; Autschbach, J. Longest-Wavelength Electronic Excitations of Linear Cyanines: The Role of Electron Delocalization and of Approximations in Time-Dependent Density Functional Theory. L. Chem. Theorv Comput. 2013, 9, 4991-5003.

[37] Oumi, M.; Maurice, D.; Lee, T. J.; Head-Gordon, M. A Diagnostic for the Applicability of the CIS and CIS(D) Excitation Energy Methods. Chem. Phys._Lett. 1997, 279, 151-157. 
[38] Rhee, Y. M.; Head-Gordon, M. Scaled Second-Order Perturbation Corrections to Configuration Interaction Singles: Efficient and Reliable Excitation Energy Methods. J. Phys. Chem. A 2007, 111, 5314-5326.

[39] Casanova, D.; Rotzinger, F. P.; Grätzel, M. Computational Study of Promising Organic Dyes for High-Performance Sensitized Solar Cells. L.Chem. Theorv Comput. 2010, 6, 12191227.

[40] Bassal, F.; Laurent, A.; Le Guennic, B.; Jacquemin, D. Exploring the Excited-States of Squarine Dyes with TD-DFT SOS-CIS(D) and ADC(2). Dyes and Pigments 2017, 138, 169-175.

[41] Fihey, A.; Favennec, A.; Le Guennic, B.; Jacquemin, D. Investigating the Properties of PODIPYs (Phosphorus-dipyrromethene) with Ab-initio Tools. Phys. Chem. Chem. Phys. 2016, $18,9358-9366$.

[42] Shao, Y. Gan, Z.; Epifanovsky, E.; et al. Advances in Molecular Quantum Chemistry contained in the Q-Chem 4 Program Package. Mol.Phvs. 2015, 113, 184-215.

[43] Chibani, S.; Le Guennic, B.; Charaf-Eddin, A.; Maury, O.; Andraud, C.; Jacquemin, D. On the Computation of Adiabatic Energies in Aza-Boron-Dipyrromethene Dyes. J. Chem. Theory Comput. 2012, 8, 3303-3313.

[44] Jacquemin, D.; Planchat, A.; Adamo, C.; Mennucci, B. TD-DFT Assessment of Functionals for Optical 0-0 Transitions in Solvated Dyes. L.Chem. Theorv Comput. 2012, 8, 2359-2372.

[45] Adamo, C.; Jacquemin, D. The Calculations of Excited-State Properties with TimeDependent Density Functional Theory. Chem.Soc.Rev. 2013, 42, 845-856. 
[46] Santoro, F.; Improta, R.; Lami, A.; Bloino, J.; Barone, V. Effective method to compute Franck-Condon integrals for optical spectra of large molecules in solution. J. Chem. Phys. 2007, $126,084509$.

[47] Santoro, F.; Lami, A.; Improta, R.; Barone, V. Effective method to compute vibrationally resolved spectra of large molecules at finite temperature in the gas phase and in solution. $J$. Chem. Phys. 2007, 126, 184102.

[48] Santoro, F.; Lami, A.; Improta, R.; Bloino, J.; Barone, V. Effective method for the computation of optical spectra of large molecules at finite temperature including the Duschinsky and Herzberg-Teller effect: The $Q_{\mathrm{x}}$ band of porphyrin as a case study. J. Chem. Phys. 2008, 128, 224311.

[49] Santoro, F. "FCclasses, a fortran 77 code": http://village.ipcf.cnr.it.

[50] Andrienko, G. http://www.chemcraftprog.com.

[51] Le Bahers, T.; Adamo, C.; Ciofini, I. A qualitative Index of Spatial Extent in Chargetransfer Excitations. L.Chem. Theorv Comput. 2011, 7, 2498-2506.

[52] Jacquemin, D.; Le Bahers, T.; Adamo, C.; Ciofini, I. What is the "best" atomic charge model to describe through-space charge-transfer excitations? Phys. Chem. Chem. Phys. 2012, 14, $5383-5388$.

[53] Mukherjee, S.; Thilagar, P. Effect of Alkyl Substituents in BODIPYs: A Comparative DFT Computational Investigation. $\underline{R S C A d v}$. 2015, 5, 2706-2714. 
[54] Zatsikha, Y. V.; Nemez, D. B.; Davis, R. L.; Singh, S.; Herbert, D. E.; King, A. J.; Ziegler, C. J.; Nemykin, V. N. Testing the Limits of the BOPHY Platform: Preparation, Characterization, and Theoretical Modeling of BOPHYs and Organometallic BOPHYs with Electron-Withdrawing Groups at $\beta$-Pyrrolic and Bridging Positions. Chem.Eur. J. 2017, 23, 14786-14796..

[55] Truc Vu, T.; Dvorko, M.; Schmidt, E. Y.; Audibert, J.-F.; Retailleau, P.; Trofimov, B. A.; Pansu, R. B.; Clavier, G.; Méallet-Renault, R. Understanding the Spectroscopic Properties and Aggregation Process of a New Emitting Boron Dipyrromethene (BODIPY). J. Phys. Chem. C 2013, $117,5373-5385$.

[56] Green, A. P.; Buckley, A. R. Solid State Concentration Quenching of Organic Fluorophores in PMMA. Phvs. Chem. Chem. Phvs. 2015, 17, 1435-1440.

[57] Bellier, Q.; Pégaz, S.; Aronica, C.; Le Guennic, B.; Andraud, C.; Maury, O. Near-Infrared Nitrofluorene Substitued Aza-Boron-dipyrromethene Dyes. Org Lett. 2011, 13, 22-25.

[58] Petrushenko, K. B.; Petrushenko, I. K.; Petrova, O. V.; Sobenina, L. N.; Trofimov, B. A. Novel Environment-Sensitive 8- $\mathrm{CF}_{3}-\mathrm{BODIPY}$ Dye with 4-(dimethylamino)phenyl Group at the 3-Position: Synthesis and Optical Properties. Dyes and Pigments, 2017, 136, 488-495.

[59] Gao, Z.; Hao, Y.; Zhen, M.; Chen, Y. A Fluorescent Dye with Large Stokes Shift and High Stability: Synthesis and Application to Live Cell Imaging. $R S C A d v$. 2017, 7, 7604-7609.

[60] Hemmer, E.; Benayas, A.; Légaré, F.; Vetrone, F. Exploiting the Biological Windows: Current Perspectives on Fluorescent Bioprobes emitting above 1000 nm. Nanoscale Horiz. 2016, $1,168-184$. 
[61] Chen, J.; Burghart, A.; Derecskei-Kovacs, A.; Burgess, K. 4,4-Difluoro-4-bora-3a,4a-diaza$s$-indacene (BODIPY) Dyes Modified for Extended Conjugation and Restricted Bond Rotations. J.Org. Chem. 2000, 65, 2900-2906.

[62] Bucher, L.; Aly, S. M.; Desbois, N.; Karsenti, P.-L.; Gros, C. P.; Harvey, P. D. Random Structural Modification of a Low-Band-Gap BODIPY-Based Polymer. J. Phys. Chem. C 2017, $121,6478-6491$.

[63] Swavey, S.; Quinn, J.; Coladipietro, M.; Cox, K. G.; Brennaman, M. K. Tuning the Photophysical Properties of BODIPY Dyes through Extended Aromatic Pyrroles. RSC Adv. 2017, 7, 173-179.

[64] Shimizu, S.; Lino, T.; Araki, Y.; Kobayashi, N. Pyrrolopyrrole aza-BODIPY Analogues: A Facile Synthesis and Intense Fluorescence. Chem. Commun. 2013, 49, 1621-1623.

[65] Li, H.-J.; Fu, W.-F.; Li, L.; Gan, X.; Mu, W.-H.; Chen, W.-Q.; Duan, X.-M.; Song, H. B. Intense One- and Two-Photon Excited Fluorescent Bis $\left(\mathrm{BF}_{2}\right)$ Core Complex Containing a 1,8Naphthyridine Derivative. Org. Lett. 2010, 12, 2924-2927. 


\section{Page 35 of 35}

TABLE OF CONTENTS (TOC) GRAPHIC

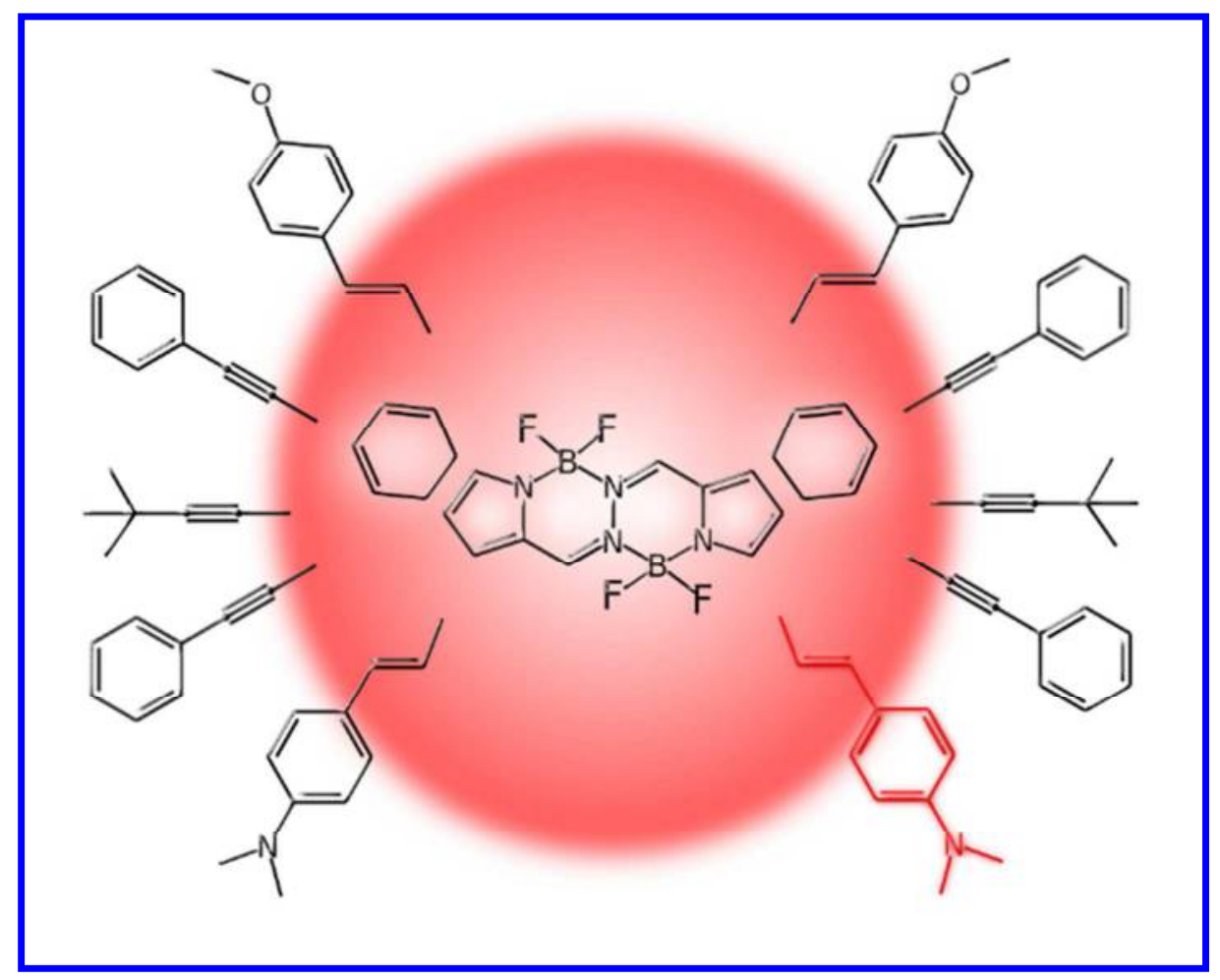

30

31

32

33

34

35

36

37

38

39

40

41

42

43

44

45

46

47

48

49

50

51

52

53

54

55

56

57

58

59

60 